\title{
Publisher's Note: Extended particle-in-cell schemes for physics in ultrastrong laser fields: Review and developments [Phys. Rev. E 92, 023305 (2015)]
}

\author{
A. Gonoskov, S. Bastrakov, E. Efimenko, A. Ilderton, M. Marklund, I. Meyerov, A. Muraviev, A. Sergeev, \\ I. Surmin, and E. Wallin \\ (Received 11 September 2015; published 17 September 2015)
}

DOI: 10.1103/PhysRevE.92.039903

PACS number(s): 02.70.-c, 52.27.Ep, 12.20.Ds, 41.75.Jv, 99.10.Fg

This paper was published online on 18 August 2015 with an omission in the Acknowledgments. The Acknowledgments should read as "The research is supported by the Ministry of Education and Science of the Russian Federation (the Agreement No. 02.B.49.21.0003 between The Ministry of Education and Science of the Russian Federation and Lobachevsky State University of Nizhni Novgorod), by the Russian Foundation for Basic Research (Projects No. 14-07-31211, No. 14-02-31495, and No. 15-37-21015), by the Olle Engkvist Foundation (Grant No. 2014/744), by the Swedish Research Council (Grants No. 2010-3727, No. 2011-4221, and No. 2012-3320) and by the Wallenberg Foundation grant "Plasma based compact ion sources." The simulations were performed on resources provided by the Swedish National Infrastructure for Computing (SNIC) and the Joint Supercomputer Center of RAS." The paper has been corrected as of 14 September 2015. The Acknowledgments are correct in the printed version of the journal. 